\title{
Focal Segmental Glomerulosclerosis in Paediatric Population of South Punjab Pakistan: A Tertiary Care Hospital Experience
}

\author{
Rabia Saleem Safdar ${ }^{1}$, M. Faisal Mehar², \\ Afsheen Asghar Khan ${ }^{3}$, Nusrat Buzdar ${ }^{4}$
}

\begin{abstract}
Objectives: To find out frequency, clinicopathological features, response of treatment and outcome among children with primary focal segmental glomerulosclerosis (FSGS).

Methods: This retrospective, non-interventional medical charts review study was conducted from a period of January 2011 to January 2020 at Pediatric Department of Nishtar Medical University Hospital, Multan, Pakistan. During the nine years study period, children of both genders, aged less than 16 years, with renal biopsies proven FSGS were included. Patient's demographic along with clinical and laboratory data, urine dipstick for proteinuria, renal functions, 24 hours urinary protein and ultrasonography findings of kidneys, ureters and bladder (KUB) were noted from case records. Response rates of various treatment options and their outcome like remission, partial remission, no remission with stable kidney disease \& no remission with progression of kidney disease were noted.

Results: During the study duration, out of 307 renal biopsies performed in glomerulonephritis cases, 124 (40.4\%) had primary FSGS. In 124 primary FSGS cases, mean age was $8.83 \pm 3.05$ years while most of the children, $70(56.5 \%)$ were above 10 years of age. Majority of the cases, $64(51.6 \%)$ were male. Mean follow up duration was noted to be $28.35+18.47$ months. Most of the cases, $68(54.8 \%)$ were found to have complete remission, $22(17.7 \%)$ partial remission while $11(8.9 \%)$ progressed to ESKD.

Conclusions: Among children, frequency of primary FSGS was high at our setting. Most of the cases achieved sustained remission rates with the help of immunosuppressive drugs. Cyclosporine and tacrolimus were found to be the most effective drugs.
\end{abstract}

KEYWORDS: Focal segmental glomerulosclerosis, Complete remission, End stage kidney disease, Immunosuppressive drugs.

doi: https://doi.org/10.12669/pjms.37.2.3535

How to cite this:

Safdar RS, Mehar MF, Khan AA, Buzdar N. Focal Segmental Glomerulosclerosis in Paediatric Population of South Punjab Pakistan: A Tertiary Care Hospital Experience. Pak J Med Sci. 2021;37(2):510-514. doi: https://doi.org/10.12669/pjms.37.2.3535

This is an Open Access article distributed under the terms of the Creative Commons Attribution License (http://creativecommons.org/licenses/by/3.0), which permits unrestricted use, distribution, and reproduction in any medium, provided the original work is properly cited.

1. Rabia Saleem Safdar, FCPS (Pediatric Medicine),

2. M. Faisal Mehar, FCPS (Pediatric Medicine),

3. Afsheen Asghar, FCPS (Pediatric Medicine),

4. Nusrat Buzdar, FCPS (Pediatric Medicine),

1-4: Department of Pediatrics, Ward Number 19,

Nishtar Medical University Hospital, Multan, Pakistan.

Correspondence:

Rabia Saleem Safdar, FCPS (Pediatric Medicine),

Assistant Professor,

Department of Pediatric Medicine,

Nishtar Medical University Hospital,

Multan, Pakistan.

E-mail: dr_rabiasaleem@live.com

* Received for Publication:

* Revision Received:

* Revision Accepted:
August 22, 2020

November 25, 2020

November 28, 2020

\section{INTRODUCTION}

Focal segmental glomerulosclerosis (FSGS) is characterized by steroid resistant nephrotic syndrome (SRNS) as well as segmental sclerosis that involve some of the glomeruli. ${ }^{1}$ Among pediatric age groups, FSGS is estimated to account for around 7 to $20 \%$ of all forms of idiopathic nephrotic syndrome (NS)., ${ }^{2,3}$ FSGS is also noted to be commonly associated with hematuria and hypertension. At earlier stages, FSGS is difficult to distinguish from minimal change disease (MCD). ${ }^{4}$ Globally, incidence of FSGS is on the rise. ${ }^{5}$ Canada reports 0.4 to 0.9 new cases per 100000 children 
annually. ${ }^{6}$ Local data from Pakistan showed FSGS to be the commonest histopathological pattern among children having SRNS. ${ }^{7}$

FSGS is considered to have variable outcomes as half of the cases are observed to reach end stage kidney disease (ESKD) when they reach 15 years of age whereas multi-centric data from developing countries noted FSGS to be the commonest form of GS contributing to $10.2 \%$ pediatric cases that ended up as ESKD. 8,9

Natural history of FSGS has been elaborated but its management is still a topic of concern. FSGS is the most common form of SRNS among children in Pakistan but scarcity of details exists at local level about its clinicopathological analysis and outcome. This is the $1^{\text {st }}$ retrospective analysis of pediatric age group having biopsy proven FSGS from South Punjab Pakistan. This study was aimed to find out frequency, clinicopathological features, response of treatment and outcome among children with primary FSGS.

\section{METHODS}

This retrospective, non-interventional medical charts review study was conducted from a period of January 2011 to January 2020 at Pediatric Department of Nishtar Medical University Hospital, Multan, Pakistan. During this nine years period, a total of 307 children of both gender, aged less than 16 years, with renal biopsies proven FSGS with the help of light microscopy (LM) and immunofluorescence (IF) were included. Out of those 307 cases, those having FSGS, with more than six months of follow up were further analyzed in this study. All children having secondary etiologies of FSGS or those who lost follow up were not included in the final analysis. Primary FSGS were the cases who are proved on biopsy as FSGS without underlying pathology. Secondary cases were those who had underlying pathology like HIV, Hepatitis, Sickle cell disease, Hemolytic ureamic syndrome etc and were excluded by clinical evaluation and laboratory evaluation. Lost to follow up were those children who lost visit for three months at any time during the study period of nine year before categorization. Approval from Institutional Ethical Committee (Ref. No. 15226, Dated: 12-08-2020) was taken for this study.

Patient's demographic along with clinical and laboratory data, urine dipstick for proteinuria, renal functions, 24 hours urinary protein and ultrasonography findings of kidneys, ureters and bladder (KUB) were noted from case records.
Disease definitions as well treatment outcomes were labeled as per "The Kidney Disease: Improving Global Outcomes" (KDIGO) 2012 guidelines. Outcome like remission, partial remission, no remission with stable kidney disease \& no remission with progression of kidney disease were followed for the period of 12 months, if remained constant during the said period, were finally considered the final outcome and added in data. Outcome as ESKD was also noted. Complete Remission was labeled as proteinuria $<4 \mathrm{mg} / \mathrm{m} 2 /$ hour or nil to trace on urine dipstick testing for three consecutive days. Partial Remission was taken as proteinuria $>4 \mathrm{mg} / \mathrm{m} 2$ / hour but $<40 \mathrm{mg} / \mathrm{m} 2 /$ hour or $1+$ to $2+$ protein on urine dipstick testing for 3 consecutive days. Relapse was labeled as proteinuria $>40 \mathrm{mg} / \mathrm{m} 2$ / hour or protein: creatinine ratio $>2$ or $\geq 3+$ protein on urine dipstick testing for three consecutive days. No remission with stable kidney disease was considered as proteinuria $>40 \mathrm{mg} / \mathrm{m} 2$ /hour or protein: creatinine ratio $>2$ or $\geq 3+$ protein on urine dipstick testing for three consecutive days along with normal renal parameters. No remission with progression of kidney disease was proteinuria $>40$ $\mathrm{mg} / \mathrm{m} 2$ / hour or protein: creatinine ratio $>2$ or $\geq 3+$ protein on urine dipstick testing for 3 consecutive days along with deranged renal parameters. End stage kidney disease was marked as chronic kidney disease with stage $\mathrm{v}$ (GFR $<15 \mathrm{ml} /$ minute/1.73m2).

Institutional standard treatment protocols as per MENDOZA treatment were used in the initial years of study. Prednisolone $60 \mathrm{mg} / \mathrm{m} 2 /$ day for duration of 4-6 weeks as standard treatment. If remission noted, dosage was reduced as $40 \mathrm{mg} /$ $\mathrm{m} 2$ /day every alternate day for further duration of 4 weeks and gradual tapering over three to six months. Pulse methyl-prednisolone was started after taking biopsy of those who did not respond to steroids over eight weeks duration according to MENDOZA protocol and cyclophosphamide was also added in steroid resistant cases. Those who didn't respond to MENDOZA protocol, were started cyclosporine and sustained remission. In last five years, 2nd line immunosuppressive drugs were used as center protocol for steroid resistant cases of FSGS. Cyclosporine was the commonest 2nd line choice as three to five $\mathrm{mg} / \mathrm{kg} /$ day in two divided dosages while keeping a target between $100-150 \mathrm{ng} / \mathrm{dL}$ in mind for a period of 24 months in cases which responded or stopped in cases that had failure in showing partial or complete response. Tacrolimus $(0.1 \mathrm{mg} / \mathrm{kg} /$ day in 2 divided dosages) and mycophenloate mofetil $(1200 \mathrm{mg} / \mathrm{m} 2 /$ day in 2 
divided dosages) were some of the other 2 nd line treatments in the later years of this study. Tapering off of drugs was done as per clinical response. SPSS (Statistical Package for Social Science USA) version 24.0 was used for data entry and analysis. Frequency and percentages were of qualitative variables were described as percentages and frequencies while quantitative variables were highlighted as mean and standard deviation.

\section{RESULTS}

During the study duration, out of 307 renal biopsies performed in GN cases, 124 (40.4\%) cases were noted to have FSGS. These 124 cases were considered for further analysis. In 124 FSGS cases, mean age was $8.83 \pm 3.05$ years while most of the children, $70(56.5 \%)$ were above 10 years of age. Majority of the cases, $64(51.6 \%)$ were male representing a male to female ratio of 1.07:1. (Table-I)

At the time of admission, microscopic hematuria was noted among 28 (22.6\%) cases. Mean red blood cell count per microliter was noted as $109.35+108$ on urinary dipstick test. Mean serum albumin was noted as $1.83 \pm 0.83 \mathrm{gm} / \mathrm{dL}$ while mean serum creatinine was recorded as $0.52 \pm 0.35 \mathrm{mg} / 100 \mathrm{~mL}$. There were $48(38.7 \%)$ cases with hypertensive. IF findings showed that $13(10.5 \%)$ cases were IgG positive, 24 (19.4\%) IgM positive, 4 (3.2\%) C3 positive while $83(66.9 \%)$ had negative IF findings.

In the $1^{\text {st }}$ four year of study period, standard treatment as intravenous methyl-prednisolone was started in all $30(24.2 \%)$ cases, out of which, $12(40.0 \%)$ got complete remission and eight $(40.0 \%)$ got partial remission. Cyclophosphamide along with methylprednisolone pulse was used in all those cases who did not respond to methyl-prednisolone therapy, out of which, 16 (53.3\%) reported relapse within two years of stopping this protocol and they were started on cyclosporine and achieved sustained remission. Those who showed cyclosporine toxicity or intolerance, were started on tacrolimum or MMF. Tacrolimus is not cost effective so was

Table-I: Characteristics of the Patients.

\begin{tabular}{lc}
\hline Total Cases having FSGS & 124 \\
\hline Gender & \\
Male & $64(51.6 \%)$ \\
Female & $60(48.4 \%)$ \\
Age (Mean \pm SD) & $8.83 \pm 3.05$ \\
Age Groups (years) & \\
$\leq 10$ & $54(43.5 \%)$ \\
$>10$ & $70(56.5 \%)$ \\
\hline
\end{tabular}

Table-II: Response of Various Drugs Used

\begin{tabular}{lc}
\hline Drugs & No. $(\%)$ \\
\hline Methyl-prednisolone pulse with & 30 \\
$\quad$ Cyclophosphamide & \\
Complete Remission & $12(40.0 \%)$ \\
Partial Remission & $8(26.7 \%)$ \\
No-remission with Stable Kidney Disease & $5(16.7 \%)$ \\
No-remission with Progressive Kidney Disease & $3(10.0 \%)$ \\
ESKD & $2(6.7 \%)$ \\
Cyclosporine & 65 \\
Complete Remission & $43(66.2 \%)$ \\
Partial Remission & $10(15.4 \%)$ \\
No-remission with Stable Kidney Disease & $5(7.7 \%)$ \\
No-remission with Progressive Kidney Disease & $2(3.1 \%)$ \\
ESKD & $5(7.7 \%)$ \\
Tacrolimus & 13 \\
Complete Remission & $9(69.2 \%)$ \\
Partial Remission & $1(7.7 \%)$ \\
No-remission with Stable Kidney Disease & $1(7.7 \%)$ \\
No-remission with Progressive Kidney Disease & $1(15.4 \%)$ \\
ESKD & $1(7.7 \%)$ \\
Mycophenloate Mofetil & 12 \\
Complete Remission & $4(33.3 \%)$ \\
Partial Remission & $3(25.0 \%)$ \\
No-remission with Stable Kidney Disease & $3(25.0 \%)$ \\
No-remission with Progressive Kidney Disease & $1(8.3 \%)$ \\
ESKD & $1(8.3 \%)$ \\
\hline
\end{tabular}

not the choice in all cases. The response rates of different drugs used is shown in table number two. There were 4 cases of familial FSGS in our cases and they were started on supportive treatment which was diuretics and angiotensin converting enzyme (ACE) inhibitors. In these familial FSGS cases, three developed ESKD out of which one expired while one remaining cases developed progressive kidney disease. The response rates of different drugs used is shown in Table-II.

Mean follow up duration was noted to be $28.35+18.47$ months. The outcome of studied cases is shown in Table-III. Most of the cases, 68 (54.8\%) were found to have complete remission, $22(17.7 \%)$ partial remission while $11(8.9 \%)$ progressed to ESKD (1 case expired, two referred to Sindh Institute of Urology and Transplantation [SUIT], Karachi for kidney transplant while eight are on dialysis at our follow up). All those cases who reported complete

Table-III: Outcome at the End of the Study Period.

\begin{tabular}{ll}
\hline Outcome & No. $(\%)$ \\
\hline Complete Remission & $68(54.8 \%)$ \\
Partial Remission & $22(17.7 \%)$ \\
No-remission with Stable Kidney Disease & $14(11.3 \%)$ \\
No-remission with Progressive & $9(5.6 \%)$ \\
$\quad$ Kidney Disease & $11(8.9 \%)$ \\
End Stage Kidney Disease & 11 \\
\hline
\end{tabular}


remission are having sustained remission up until the end of the study period.

\section{DISCUSSION}

During nine years of study period, among renal biopsies performed in GN cases, $40.4 \%$ were noted to have FSGS. Spectrum of GN has been found varying across different parts of the world. From Croatia, Batanic et al. ${ }^{10}$ noted very similar findings where they noted FSGS to be among 24.6\% GN cases followed by Mesangioproliferative glomerulonephritis (MesPGN) in 19.2\%. A study from Bangladesh ${ }^{11}$ found MesPGN to be the commonest histological finding among children with GN. From Pakistan, MCD followed by FSGS has been found to be the most common histopathological findings in GN in a previous research. ${ }^{12}$ These variations could be because of differences subjected to biopsy indications as well as patient referrals and racial predispositions among different nephropathies. ${ }^{13,14}$ No standard guideline exists regarding kidney biopsies among pediatric population.

Although clinicopathological aspects of FSGS have been well elaborated in the last few decades since its $1^{\text {st }}$ description by Rich in 1957, management of FSGS has always been challenging and a topic that has been debated over the years. ${ }^{14,15}$ Corticosteroids have been the mainstay of FSGS treatment while response of steroid therapy is considered to be the finest predictor of long-term outcome and prognosis. In the study, initially, steroids were used to treat FSGS children. Cyclophosphamide was added later those cases which did not respond to steroid treatment alone. It has been well established from the past findings that long term usage of steroids among SDNS children is associated with increased exposure to steroid related toxicity like osteoporosis, growth disorders, cataract, diabetes mellitus as well as psycho-emotional disorders. ${ }^{14,16}$ Various immunosuppressive drugs are supplemented with steroids commonly to avoid steroid toxicity. Our approach was quite similar to the one that has been described previously. ${ }^{14,17}$ In the current study, $40 \%$ of the children achieved complete remission and $26.7 \%$ achieved partial remission with the administration of methylprednisolone pulse along with cyclophosphamide. International literature showed complete remission rate of cyclophosphamide to be $51 \%$ while partial remission noted in $23 \%$ and no response in $26 \% .{ }^{18}$ Higher rates of complete remission as $69.4 \%$ have been reported in the past by local researcher with cyclophosphamide. ${ }^{14}$ Complete remission among $33.3 \%$ children was noted by Brazilian researchers using a combination of steroid and cyclophosphamide. $^{19}$ It was also noted in the present study that $53.3 \%$ of the cases using this combination therapy reported relapse within two years of stopping this protocol and they were started on cyclosporine and achieved sustained remission. Previous local data also found $36 \%$ of the patients using cyclophosphamide and steroid to report relapse later and were shifted to cyclosporine who achieved sustained remission. ${ }^{14}$

In FSGS, cyclosporine has been seen to minimize the relapse rates by $80 \% .^{20}$ In the present study, cyclosporine was found to be the most effective drug, achieving complete remission in $66.2 \%$ cases while $15.4 \%$ had partial remission and $7.7 \%$ were reported with ESKD. Previously published local study found complete remission as $52.6 \%$ cases while no response was seen in $12.2 \%{ }^{14}$ Researchers from other parts of the world ${ }^{21}$ have also noted cyclosporine to produce complete remission rates of $44.4 \%$ while data from the US found it much higher as $87 \%$ among SRNS patients..$^{22}$ Difference in standardization as well as duration could be the one major reason for this variability in response with cyclosporine in children with FSGS. Dependency upon cyclosporine is another aspect that needs to be considered as is the cases with steroid therapy.

Tacrolimus was administered in 13 cases which were resistant to cyclosporine and complete remission was noted in $69.2 \%$ of these cases. Recently, tacrolimus has been recommended as an alternative choice to cyclosporine for SRNS and has been shown to be well tolerated showing a complete remission rate of as high as $81 \% \cdot 15,22,23$ MFM was used instead of tacrolimus in 12 cases while complete remission among $33.3 \%$ and partial remission in $25 \%$ cases were reported which is quite similar to previously published studies. ${ }^{22,24}$

In the present study $5.6 \%$ cases had progression in their kidney disease and $8.9 \%$ ended up in ESKD. Worldwide literature present wide variability of 13$78 \%$ cases ending up as ESKD in a follow up period of as long as 20 years which again emphasizes the variability in treatment approaches in children having FSGS., 74,16

Strength and Limitations of the study: Uniformity in the diagnostic and histopathological approach as good sample size and long duration of follow up are some of the strengths of this study. Selection bias among the study participants could be a limitation, as is usually the case among 
biopsy base researchers. We also could not treat all the cases as per a single standard protocol over the years which can also present difference in outcomes regarding different therapeutic options. Comparatively shorter duration of follow up is another limitation of this study.

\section{CONCLUSIONS}

Among children, frequency of primary FSGS was high at our setting. Most of the cases achieved sustained remission rates with the help of immunosuppressive drugs. Cyclosporine and tacrolimus were found to be the most effective drugs.

Acknowledgements: The authors are thankful to Prof. Dr. Fauzia Zafar, Chairperson \& HOD Pediatrics, Nishtar Medical University Hospital, Multan, for her valuable guidance and support in completion of this research.

\section{Grant Support \& Financial Disclosure: None. Conflict of Interest: None.}

\section{REFERENCES}

1. Rosenberg AZ,KoppJB.FocalSegmentalGlomerulosclerosis. Clin J Am Soc Nephrol. 2017;12(3):502-517. doi: 10.2215/ CJN.05960616

2. Schnaper HW. Idiopathic focal segmental glomerulosclerosis. Semin Nephrol. 2003;23:183-193. doi: 10.1053/snep.2003.50016

3. Srivastava T, Simon SD, Alon US. High incidence of focal segmental glomerulosclerosis in nephrotic syndrome of childhood. Pediatr Nephrol. 1999;13:13-18. doi: 10.1007/ s004670050555

4. Almardini RI, Albaramki JH, Al-Saliata GM, Farah MQ, AlRabadi JG, Albderat JT. Pediatric Focal Segmental Glomerulosclerosis in Jordan: A Tertiary Hospital Experience. Saudi J Kidney Dis Transpl. 2018;29(4):816-821. doi: 10.4103/1319-2442.239655

5. Guruswamy Sangameswaran KD, Baradhi KM. Focal Segmental Glomerulosclerosis. [Updated 2019 Dec 20]. In: StatPearls [Internet]. Treasure Island (FL): StatPearls Publishing; 2020. Available from: https://www.ncbi.nlm. nih.gov/books/NBK532272/

6. Filler G, Young E, Geier P, Carpenter B, Drukker A, Feber J. Is there really an increase in non-minimal change nephrotic syndrome in children? Am J Kidney Dis. 2003;42:1107-1113. doi: 10.1053/j.ajkd.2003.08.010

7. Sadaf A, Khemchand MN, Fouzia L, Asia Z Clinicopathological Profile of Pediatric Renal Biopsies at a Tertiary Care Hospital, Pakistan. Saudi J Kidney Dis Transpl. 2018;29(6):1403-1409. doi: 10.4103/1319-2442.248290

8. Zagury A, Oliveira AL, Montalvao JA, Novaes RH, Vinicius $\mathrm{M}$, de Moraes CA, et al. Steroid-resistant idiopathic nephrotic syndrome in children: Long-term follow-up and risk factors for end-stage renal disease. J Bras Nefrol. 2013;35:191-199. doi: 10.5935/0101-2800.20130031

9. Mekahli D, Liutkus A, Ranchin B, Yu A, Bessenay L, Girardin E, et al. Long-term outcome of idiopathic steroidresistant nephrotic syndrome: A multicenter study. Pediatr Nephrol 2009;24:1525-1532. doi: 10.1007/s00467-009-1138-5
10. Batini D, Scukanec-Spoljar M, Milosevic D, Subat-Dezulovic M, Saraga M, Delmis J, et al. Clinical and histopathological characteristics of biopsy-proven renal diseases in Croatia. Acta Med Croatica. 2007;61(4):361-364.

11. Begum A, Santa SM, Al Mamun A, Jesmin T, Huque SS, Roy RR. Renal histopathological profile of Bangladeshi children in a tertiary care hospital. Asian J Pediatr Nephrol. 2019;2:104-105. doi: 10.4103/AJPN.AJPN_20_19

12. Nasir H, Chaudhry S, Raza W, Moatasim A, Mamoon N, Akhtar N. Role of immunofluorescence in the diagnosis of glomerulonephritis. J Pak Med Assoc. 2012;62;240-243.

13. Jiang $\mathrm{M}, \mathrm{Xiao} \mathrm{Z}$, Rong $\mathrm{L}, \mathrm{Xu} \mathrm{Y}$, Chen L, Mo Y, et al. Twenty-eight-year review of childhood renal diseases from renal biopsy data: A single centre in China. Nephrology. 2016;21:1003-1009. doi: 10.1111/nep.12702

14. Lanewala A, Mubarak M, Kazi JI, Akhter J, Sher A, Fayyaz A, et al. A Clinicopathologic Study of Primary Focal Segmental Glomerulosclerosis in Children. Saudi J Kidney Dis Transpl. 2012;23(3):513-520

15. Sethna CB, Gipson DS. Treatment of FSGS in Children. Adv Chronic Kidney Dis. 2014;21(2):194-199. doi: 10.1053/j. ackd.2014.01.010

16. Chitalia VC, Wells JE, Robson RA, Searle M, Lynn KL. Predicting renal survival in primary focal segmental glomerulosclerosis from the time of presentation. Kidney Int. 1999;56:2236-2242. doi: 10.1038/sj.ki.4491164

17. Han $\mathrm{KH}, \mathrm{Kim} \mathrm{SH}$. Recent advances in treatments of primary focal segmental glomerulosclerosis in children. Biomed Res Int. 2016;2016:3053706. doi: 10.1155/2016/3053706

18. Korbet SM. Treatment of primary focal segmental glomerulosclerosis. Kidney Int. 2002;62:2301-2310.

19. Martinelli R, Pereira LJ, Silva OM, Okumura AS, Rocha $\mathrm{H}$. Cyclophosphamide in the treatment of focal segmental glomerulosclerosis. Braz J Med Biol Res. 2004;37(9):13651372. doi: 10.1590/s0100-879x2004000900011

20. Niaudet $P$, Habib R. Cyclosporine in the treatment of idiopathic nephrosis. J Am Soc Nephrol. 1994;5(4):10491056.

21. Samuel S, Bitzan M, Zappitelli M, Dart A, Mammen C, Maury $\mathrm{P}$, et al. Canadian society of nephrology commentary on the 2012 KDIGO clinical practice guideline for glomerulonephritis: management of nephrotic syndrome in children. Am J Kidney Dis. 2014;63(3):354-362. doi: 10.1053/j.ajkd.2013.12.002

22. Loeffler K, Gowrishanker M, Yiu V. Tacrolimus therapy in pediatric patients with treatment resistant nephrotic syndrome. Pediatr Nephrol. 2004;19:281-287. doi: 10.1007/ s00467-003-1370-3

23. Jahan A, Prabha R, Chaturvedi S, Mathew B, Fleming D, Agarwal I. Clinical efficacy and pharmacokinetics of tacrolimus in children with steroid-resistant nephrotic syndrome. Pediatr Nephrol. 2015;30(11):1961-1967. doi: 10.1007/s00467-015-3133-3

24. Gulati A, Sinha A, Gupta A, Dinda A, Hari P, Bagga A. Treatment with tacrolimus and prednisolone is preferable to intravenous cyclophosphamide as the initial therapy for children with steroid-resistant nephrotic syndrome. Kidney Int. 2012;82(10):1130-1135. doi: 10.1038/ki.2012.238

\section{Authors' Contribution:}

RSS: Data Analysis, Drafting, responsible for data's authenticity and integrity.

MFM: Literature Review, Introduction.

AA: Data Interpretation, Proof Reading.

NB: Literature Review, Discussion. 